"El saber de mis hijos

hará mi grandeza"

Año 13.

Núm. 33

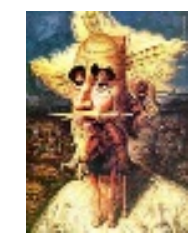

Revista de Investigación

Académica sin Frontera

ISSN: 2007-8870

https://revistainvestigacionacademicasinfrontera.unison.mx/index.php/RDIASF

Recibido el 20 de junio de 2020. Dictaminado mediante arbitraje favorablemente 18 de diciembre de 2020.

\title{
Mesa de trabajo para prueba de protocolos de localización IoT en aplicaciones didácticas.
}

IoT test bed for localization protocols in educational applications

Dr. Joel Ruiz Ibarra

https://orcid.org/0000-0002-4932-2006

Mtro. Juan Pablo Aguilar Limón

https://orcid.org/0000-0003-1811-9803

M.C. Edgar Alberto Espinoza Zallas https://orcid.org/0000-0001-7667-5782

M.C. Patricia Andrade Salinas https://orcid.org/0000-0002-9723-3964

\section{Resumen}

Históricamente la tecnología ha facilitado la vida del ser humano. Con la llegada de un dispositivo mejorado o un protocolo optimizado, suele llegar un nuevo paradigma tecnológico que cambia el concepto y percepción de la propia tecnología. El Internet de las Cosas (IoT) es esta nueva corriente de dispositivos interconectados, que consigo traen un nuevo conjunto de beneficios y retos. Entre los beneficios se encuentran que se puede obtener una vasta información de los objetos que antes solo cumplían una función como una puerta, una silla, etc. Entre los retos se encuentra que se puede obtiene una vasta información de los objetos que conectamos a internet con IoT y procesar esa información se ha vuelto una tarea colosal. En este trabajo se bosqueja una mesa de pruebas para IoT con tecnología a la mano de cualquier internauta, de modo que pueda aplicarse a situaciones didácticas en el estudio de las materias de ingeniería en una universidad.

Palabras Clave: Internet de las cosas (IoT), protocolo de comunicación. 


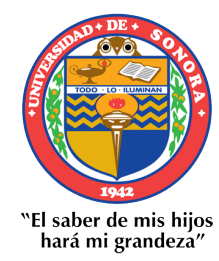

( Julio - Diciembre 2020)

Año 13.

Núm. 33

\section{Nitm. 33}

Revista de Investigación

Académica sin Frontera

ISSN: 2007-8870

Recibido el 20 de junio de 2020. Dictaminado mediante arbitraje favorablemente 18 de diciembre de 2020.

\begin{abstract}
Since its beginnings, technology has been a helpful tool for humanity, making life easier. When a new improved device or an optimized protocol is released, it comes with a new technological paradigm, changing the way we conceive it. Now-a-days Internet of Things (IoT) is a new connectivity paradigm which connects everything to internet. That new paradigm produces a huge amount of data. Processing that amount of data is a challenge. This proposal includes a IoT framework for educational applications, useful as a learning object for Universities.
\end{abstract}

Keywords: Internet of things, comunication protocol

Introducción

Las WSN por sus siglas en inglés (Wireless Sensor Network), se tratan de redes de dimensiones locales, con la capacidad de agruparse en torno a un sumidero o "Sink" el cual asocia a su identificador de red para administrar la información entregada por los mismos.

A este tipo de redes también se les conoce como Redes de área personal (WPAN) la cual se define como una red computacional centrada en el usuario que le permite comunicarse con sus dispositivos cercanos. (S. C. Mukhopadhyay y H. Leung 2010).

Estas redes cubren normalmente un rango cercano a pocos metros, ya sea para uso personal o entornos o ambientes de trabajo.

Los conceptos de redes centradas en el usuario se desarrollaron a partir de una idea del Instituto Tecnológico de Massachusetts; (MIT, por sus siglas en inglés.) la cual, en 1995 planteaba usar las corrientes eléctricas internas del cuerpo humano para la comunicación entre dispositivos implantados en el mismo. Fue aceptado como concepto por IBM Research. Posteriormente, experimentó muchas variantes desarrolladas por diferentes instituciones de investigación o compañías. La diferente variedad de soluciones PAN incluyen:

- Proyecto Oxygen (MIT)

- Asociación infrarroja de datos (IrDa, por sus siglas en inglés.)

- Bluetooth

- IEEE 802.15 
"El saber de mis hijos

hará mi grandeza"

Año 13.

Núm. 33
Revista de Investigación

Académica sin Frontera

ISSN: 2007-8870

\section{https://revistainvestigacionacademicasinfrontera.unison.mx/index.php/RDIASF}

Recibido el 20 de junio de 2020. Dictaminado mediante arbitraje favorablemente 18 de diciembre de 2020.

\section{Estándar IEEE 802.15.4.}

El estándar 802.15.4 especifica la capa física y el control de acceso al medio para dispositivos inalámbricos de redes de área personal de baja intensidad (LR-WPAN). Actualmente se sigue desarrollando y manteniendo por el grupo de trabajo 802.15. Este pretende ofrecer las capas inferiores fundamentales (Física y de Control de acceso al medio) a WPAN las cuales se enfocan en comunicación de bajas tasas de intercambio, y de bajo costo para dispositivos, esto con el objetivo de aminorar el consumo de energía de estos dispositivos. (IEEE Standard 802.15.4-2011, 2011). (Alharthi, Ameera \& Morgan, Ahmed \& Semeia, Abdellatif 2020).

El grupo de trabajo 4 (TG4) del estándar IEEE 802.15, propone las siguientes especificaciones para dispositivos enfocados a WPAN:

- Tasas de transmisión de 250 kbps, 40 kbps y 20 kbps

- Dos modos de direccionamiento; 16 y 64 bits

- Soporte para dispositivos dependientes de latencia, por ejemplo, Joysticks o mandos.

- Acceso al canal tipo CSMA-CA

- Establecimiento automático de una red vía el nodo coordinador.

- Administración de potencia para asegurar un bajo consumo de energía

En la figura 1 se muestra la trama de control de capa MAC en IEEE 802.15.4

\begin{tabular}{|c|c|c|c|c|c|c|c|c|}
\hline $\begin{array}{c}\text { Bits: } \\
0-2\end{array}$ & 3 & 4 & 5 & 6 & $7-9$ & $10-11$ & $12-13$ & $14-15$ \\
\hline $\begin{array}{l}\text { Frame } \\
\text { Type }\end{array}$ & $\begin{array}{l}\text { Security } \\
\text { Enabled }\end{array}$ & $\begin{array}{l}\text { Frame } \\
\text { Pending }\end{array}$ & $\begin{array}{l}\text { Ack. } \\
\text { Request }\end{array}$ & $\begin{array}{l}\text { PAN ID } \\
\text { Compression }\end{array}$ & Reserved & $\begin{array}{l}\text { Dest. } \\
\text { Addressing } \\
\text { Mode }\end{array}$ & $\begin{array}{l}\text { Frame } \\
\text { Version }\end{array}$ & $\begin{array}{l}\text { Source } \\
\text { Addressing } \\
\text { Mode }\end{array}$ \\
\hline
\end{tabular}

Figura 1.- Trama IEEE 802.15.4

\section{Zigbee.}

Zigbee define un conjunto de protocolos de comunicación para redes de baja tasa de transferencia de datos, la cual, opta por tener como base, el estándar 802.15.4. Le otorga las 


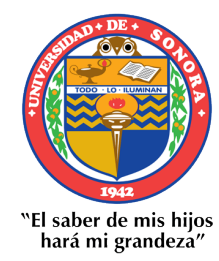

( Julio - Diciembre 2020)

Año 13.

Núm. 33

https://re

Recibido el 20 de junio de 2020. Dictaminado mediante arbitraje favorablemente 18 de diciembre de 2020.

capas físicas y de control de acceso al medio, mientras que Zigbee provee las capas superiores, refiriéndonos al modelo OSI.

Su propósito general es crear una topología de red para permitir un determinado número de dispositivos el comunicarse entre ellos y proveer funcionalidades extras como autentificación, encriptación y asociación. Zigbee es el producto de la asociación de varias compañías que formaron la alianza Zigbee, para obtener un estándar de comunicación cuyo punto fuerte es la estandarización misma, permitiendo que interfaces de diferentes fabricantes, puedan comunicarse entre ellas.

Zigbee contempla tres tipos de nodos. Un nodo coordinador que es quien levanta la red y la inicializa. Uno o varios nodos enrutadores que se encargan de retransmitir la información, empleando una versión reducida del protocolo AODV. Y uno o varios nodos finales, que se encargan de caracterizar sensores de monitoreo y enviar esa información a cualquier parte de la red a través de los nodos enrutadores. En la figura 3 se muestra una topología árbol formada por dispositivos ZigBee y el estándar IEEE 802.15.4 están enfocados a trabajar bajas tasas

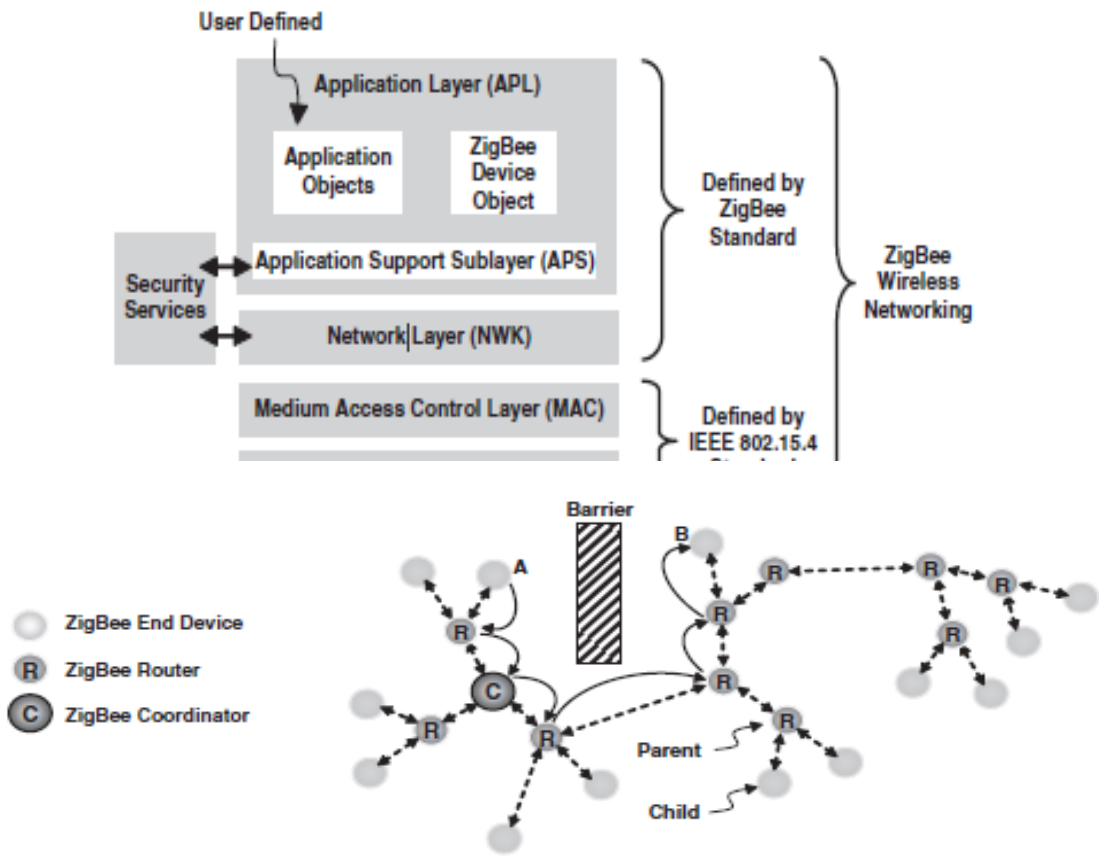

Figura 3.- Topología árbol, nodos ZigBee. 


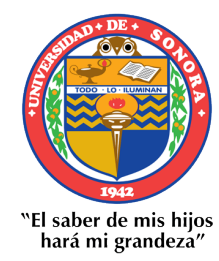

( Julio - Diciembre 2020)

Año 13.

Núm. 33

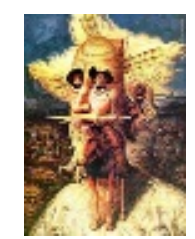

Revista de Investigación Académica sin Frontera

ISSN: 2007-8870 https://revistainvestigacionacademicasinfrontera.unison.mx/index.php/RDIASF

Recibido el 20 de junio de 2020. Dictaminado mediante arbitraje favorablemente 18 de diciembre de 2020.

de transferencia y latencias de datos muy altas, para un consumo muy bajo de energía. A pesar de ello o incluso a favor de ello, esto genera un nicho importante en aplicaciones de telemetría. Si bien internet concibe que, a mayor velocidad, mejor servicio, este no es el caso para WSN ya que es a expensas de consumo de energía que en muchas aplicaciones es limitado. IoT considera conexiones completas a internet. Conexiones al ancho de banda que tiene normalmente una $\mathrm{PC}$, lo que asume pocas o nulas restricciones de energía.

Un marco de trabajo para desarrollo de aplicaciones embebidas suele tener una arquitectura Harvard, como Arduino, NodeMCU, Pic, etc. Sin embargo, tiene gran ventaja que funcione sobre una topología modular; una arquitectura similar a Von Newmann le permite aprovechar las virtudes de diferentes transceptores trabajando sobre la misma lógica de protocolo de coordinación. La comunicación hacia los transceptores se puede dar por medio de los diferentes buses disponibles en la actualidad (UART, RS232, SPI, I2C). El módulo ZigBee, si bien es un sistema embebido muy completo, con puertos GPIO, también cuenta con un puerto RS232 para su comunicación como se muestra en la figura 4.

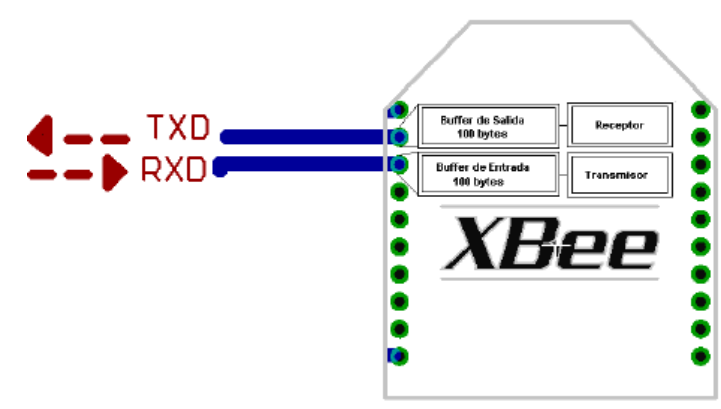

Figura 4.- Puerto de comunicación serial Xbee.

\section{WiFi en IoT}

Diversos sistemas embebidos han incorporado a sus circuitos interfaces WiFi. Esta interfaz no es la más eficiente en consumo de energía para nodos alimentados a batería, por lo que se debe considerar al momento de diseñar la aplicación del sistema. Por lo general esto no es problema si la "cosa" a la que se le va a conectar a internet está en un ambiente de interiores, ya sea hogar, oficina o industria, y cuenta con alimentación al servicio eléctrico federal. Como podemos ver, los nichos de aplicación varían y se cuenta con tecnología para abarcar una amplia gama de ellos, por lo que es importante la modularidad de la propuesta de 


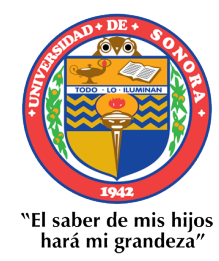

( Julio - Diciembre 2020)

Año 13.

Núm. 33

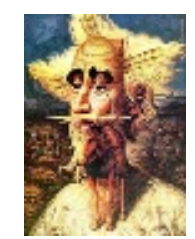

Revista de Investigación Académica sin Frontera

ISSN: 2007-8870

\section{https://revistainvestigacionacademicasinfrontera.unison.mx/index.php/RDIASF}

Recibido el 20 de junio de 2020. Dictaminado mediante arbitraje favorablemente 18 de diciembre de 2020.

coordinación para que funcione con diferentes transceptores en esta amplia gama de escenarios.

Uno de los circuitos embebidos más populares recientemente entre los entusiastas IoT y algunas compañías de desarrollo es la familia ESP. (ESP 8266, ESP32). Cuenta con un procesador RISC de 32 bits a $80 \mathrm{Mhz}$. Algo similar a un equipo de cómputo personal de finales de los $80 \mathrm{~s}$. También cuenta con puertos de entrada y salida de uso general (GPIO) y de comunicaciones SPI e I2C entre otras características particulares. (Yogendra Singh Parihar 2019) (Homera Durani 2018),

La característica más notable de un ESP8266 es que cuenta con un stack TCP/IP completo que le permite conectarse a redes WiFi y es de bajo costo (del orden de $\$ 100$ pesos mexicanos)

En la figura 5 se muestra un módulo ESP8266 (izquierda) y un módulo NodeMCU (derecha) que contiene un ESP8266 con diferentes puertos expuestos para su uso y comunicación USB.
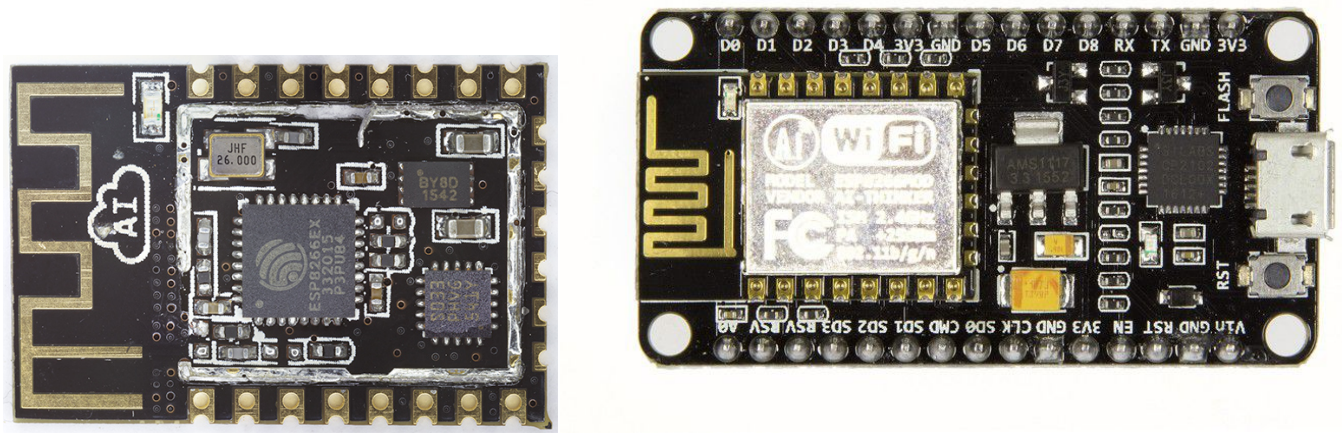

Figura 5.- Módulo ESP8266 (izquierda) y módulo NodeMCU (derecha)

Para la mesa de trabajo que aquí se propone se emplea el módulo NodeMCU con programación "Over the air" que facilita actualizar su software una vez instalado en la 
"El saber de mis hijos

hará mi grandeza"

Año 13.

Núm. 33
Revista de Investigación

Académica sin Frontera

ISSN: 2007-8870

https://revistainvestigacionacademicasinfrontera.unison.mx/index.php/RDIASF

Recibido el 20 de junio de 2020. Dictaminado mediante arbitraje favorablemente 18 de diciembre de 2020.

aplicación que le corresponde (Quadri, S. \& Sidek, Othman 2014) (Maia, Guilherme \& Guidoni, Daniel \& Aquino, Andre \& Loureiro, Antonio 2009), (Galache, J.A. \& Sotres, Pablo \& Santana, Juan \& Gutierrez, Veronica \& Sánchez, Luis \& Munoz, Luis 2013).

ESP8266 puede trabajar tanto como punto de acceso WiFi o como nodo dentro de una red, incluso puede funcionar como servidor web de recursos limitados.

La ecuación de Friis determina que hay una relación entre la disipación de la potencia de una transmisión y la distancia que ha recorrido. Esta ecuación está desarrollada para ambientes en espacio abierto donde hay visibilidad directa entre transmisor y receptor, además se desprecian las múltiples trayectorias. Es una buena primera aproximación a la trilateración que se puede realizar para encontrar un nodo con base en las potencias de señal recibida. Posteriormente debe irse corrigiendo la ubicación hasta lograr un nivel de error aceptable para la aplicación. En la figura 6 se observa el concepto fundamental de trilateración.

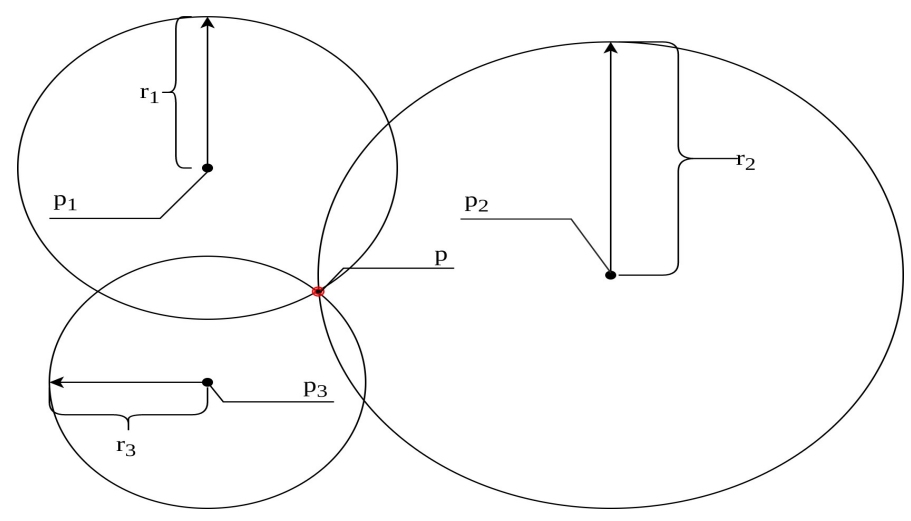

Figura 6.- Trilateración.

En la figura 6 se observa un punto rojo $\mathrm{P}$ que deseamos localizar y 3 puntos de referencia o balizas P1, P2 y P3 con coordenadas conocidas. Si la potencia de señal recibida (RSSI) es directamente proporcional a la distancia recorrida, ésta puede determinar el radio r1, r2 y r3 


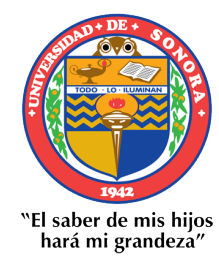

( Julio - Diciembre 2020)

Año 13.

Núm. 33

\section{.}

Recibido el 20 de junio de 2020. Dictaminado mediante arbitraje favorablemente 18 de diciembre de 2020.

de la figura 6 y por trilateración encontrar las coordenadas del punto P. En este escenario, la distancia hacia el punto $\mathrm{P}$, desde cualquier punto $\mathrm{Pn}$, se da por la ecuación 1.

$$
D=\sqrt{\left(x_{1}-x_{2}\right)^{2}+\left(y_{1}-y_{2}\right)^{2}}
$$

En un escenario ideal esto sería posible sin mayores complicaciones, sin embargo, existen diferentes factores que afectan la RSSI como son las múltiples trayectorias que generan interferencias destructivas e interferencias sumativas en diferentes puntos de la trayectoria. Además, se tienen atenuaciones por límites de frontera con diferentes materiales que se pudiera interpretar erróneamente como mayor distancia de propagación. La ecuación 2 describe la ecuación de Friis.

$$
\frac{P_{R}}{P_{T}}=\frac{G_{T} G_{R} \lambda^{2}}{16 \pi^{2} r^{2}}\left\{\begin{array}{l}
P_{R}=\text { potencia recibida, en } \mathrm{W} . \\
P_{T}=\text { potencia transmitida, en } \mathrm{W} . \\
G_{T}=\text { ganancia de la antena transmisora. } \\
G_{R}=\text { ganancia de la antena receptora. } \\
\lambda=\text { longitud de onda de la onda, en } \mathrm{m} . \\
r=\text { distancia radial entre antenas, en } \mathrm{m} .
\end{array}\right.
$$

Debido a esta discrepancia entre la ecuación de Friss y las distancias entre nodos, se propone un mapa de potencias de señales recibidas en el área de interés en el que se puede localizar el punto P. Este mapa consiste en tomar medidas de RSSI ordenadamente en una cuadrícula que abarque el área de interés donde se desea encontrar el nodo perdido, a priori del proceso de localización. Cuando se desea localizar a un nodo en esta área, se toman lecturas de las RSSI que escucha ese nodo y se consulta el mapa de potencias recibidas en esa área. De esta manera se calcula la posición por máxima verosimilitud. Este estimador es consistente si el número de observaciones $\mathrm{n}$, tiende a infinito, convergiendo en el valor verdadero de posición.

En la figura 7 se muestra un segmento de ondas electromagnéticas incidentes en un punto (abajo), donde se puede ver que suceden interferencias sumativas y destructivas entre ellas. Lo que el receptor detecta es la suma algebraica de estas señales incidentes en el punto de la 


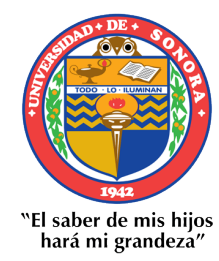

Año 13.

Núm. 33

https://revis
( Julio - Diciembre 2020)

Revista de Investigación

Académica sin Frontera

ISSN: 2007-8870

Recibido el 20 de junio de 2020. Dictaminado mediante arbitraje favorablemente 18 de diciembre de 2020.

antena receptora. En la misma figura 7 (arriba) se observan las RSSI medidas en la biblioteca de la Universidad Estatal de Sonora, a lo largo de $24 \mathrm{~m}$ desde el nodo baliza \#3. Se puede observar una variación del RSSI donde aún para distancias ligeramente mayores (punto B), el RSSI es superior que, para distancias inferiores (punto A), pero la tendencia general es a ir bajando conforme aumenta la distancia de recepción. Esto se debe al entorno, multitrayectorias, sumas y restas de ondas electromagnéticas con fases constructivas y/o destructivas. 
Año 13.

Académica sin Frontera

Núm. 33

ISSN: 2007-8870

https://revistainvestigacionacademicasinfrontera.unison.mx/index.php/RDIASF

Recibido el 20 de junio de 2020. Dictaminado mediante arbitraje favorablemente 18 de diciembre de 2020.
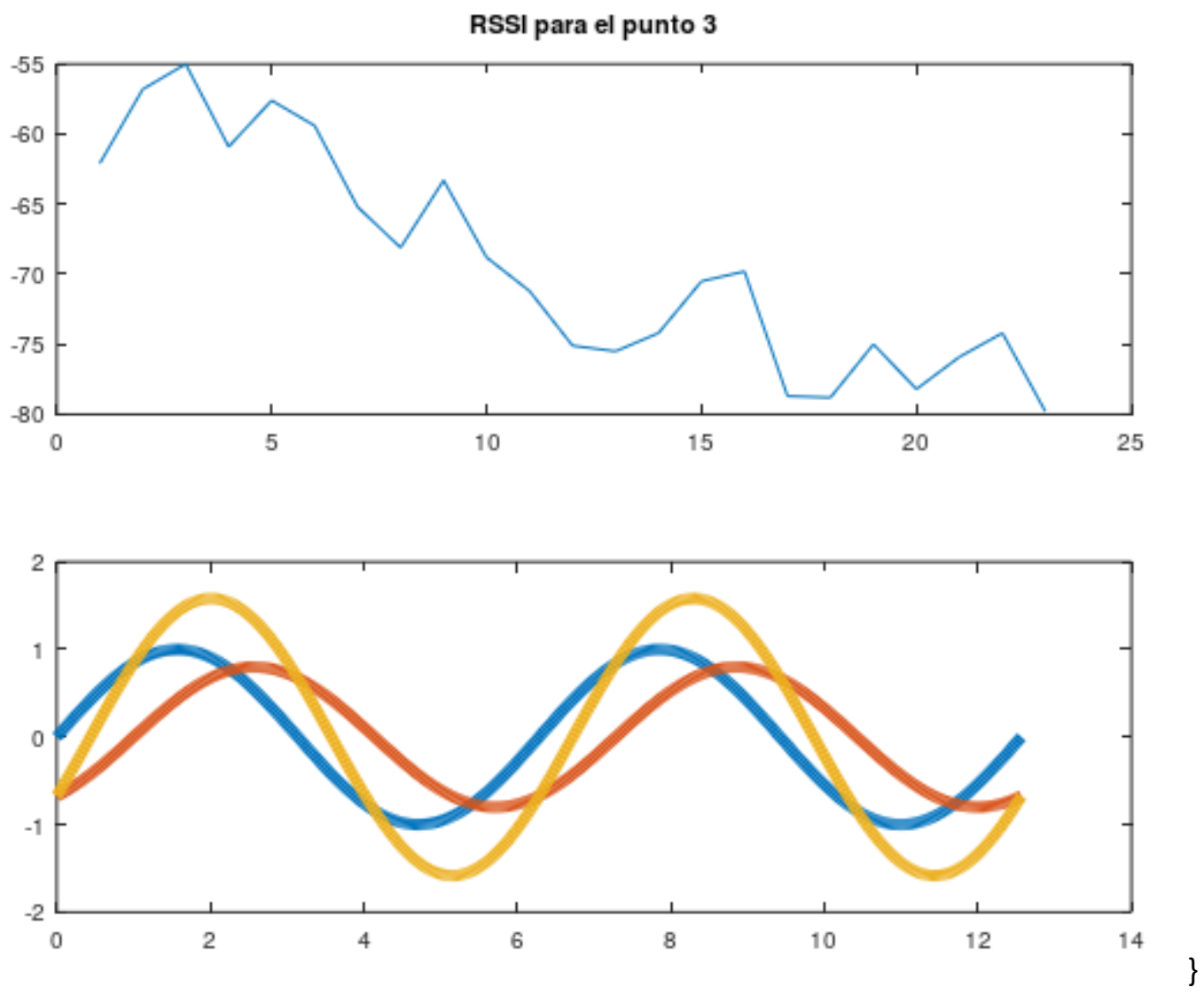

Figura 7.- RSSI baliza 3, Biblioteca Universidad Estatal de Sonora.

Para analizar la varianza de las mediciones, se realizaron varias mediciones para las mismas distancias generando la gráfica de la figura 8. Normalizando los datos y excluyendo los extremos fuera de un intervalo de confianza del $90 \%$ (como el punto 1 de la gráfica 3), se observa una variación en el peor de los casos de $10 \mathrm{~dB}$. Esta variación nos permite identificar tres puntos de ubicación en un espacio de $24 \mathrm{~m}$, por lo que sólo con la ecuación de Friis, este mecanismo nos permitiría una localización con un error de $8 \mathrm{~m}$. 
"El saber de mis hijos

hará mi grandeza"

Año 13.

Núm. 33
Revista de Investigación

Académica sin Frontera

ISSN: 2007-8870

Recibido el 20 de junio de 2020. Dictaminado mediante arbitraje favorablemente 18 de diciembre de 2020.
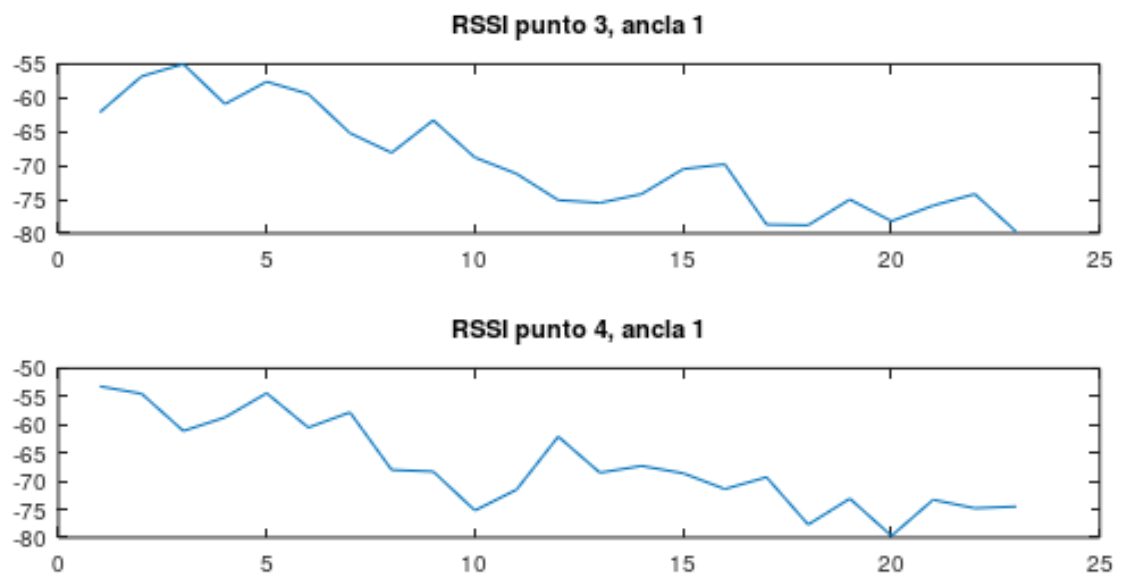

RSSI punto 5, ancla 1

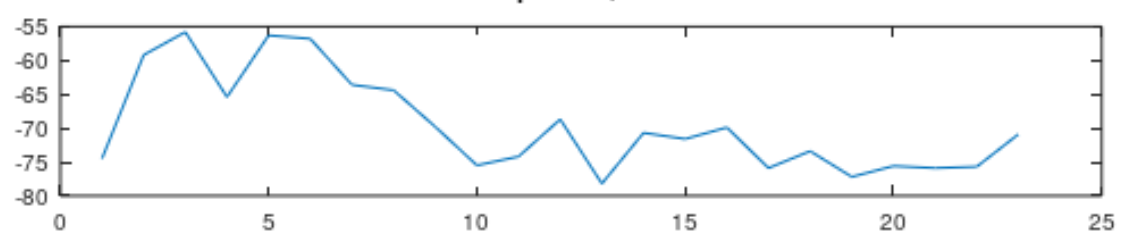

Figura 8.- Tres mediciones de RSSI sobre las mismas distancias.

Esta misma información, extraída de al menos otros dos nodos balizas posicionados en los

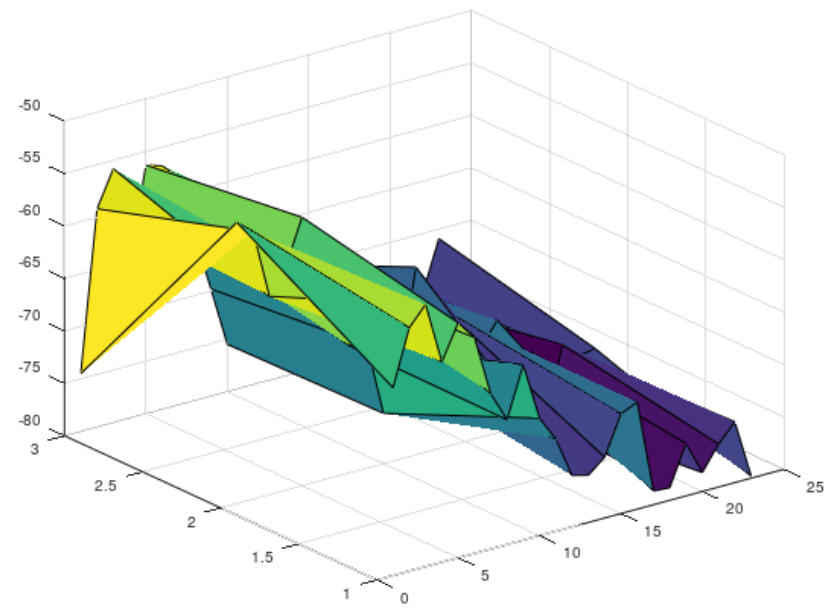

Figura 9.- Mapa de RSSI para baliza 3 en 25 metros lineales. 


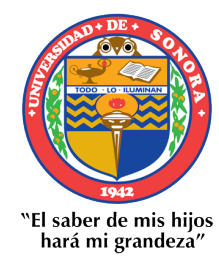

Año 13.

Núm. 33
( Julio - Diciembre 2020)

Revista de Investigación

Académica sin Frontera

ISSN: 2007-8870

https://revistainvestigacionacademicasinfrontera.unison.mx/index.php/RDIASF

Recibido el 20 de junio de 2020. Dictaminado mediante arbitraje favorablemente 18 de diciembre de 2020.

extremos del recinto donde aún tenga cobertura WiFi a un solo salto, mejora el algoritmo de localización. Ya que se coteja el RSSI de cada una de las anclas que, aunque la ecuación de Friis denote una distancia errónea, ésta se corrige con las lecturas de las otras n-balizas y la correlación con el mapa de RSSI generado a priori. En la figura 8 se muestra el mapa de coberturas RSSI para una misma ancla (baliza) en el espacio de búsqueda abordado.

Se considera como trabajo futuro realizar este experimento con diferentes radio-transmisores, comparando el grado de precisión y exactitud y la pertinencia para diferentes aplicaciones de localización de activos y personal de la Universidad Estatal de Sonora.

\section{Conclusiones.}

Se logró demostrar la relación entre potencia de señal recibida (RSSI) y la distancia de recepción de la señal. Se observó que varía con respecto al entorno y número de superficies donde rebota la señal y que un ambiente abierto sin superficies intermedias es el escenario óptimo.

Bibliografía.

Alharthi, Ameera \& Morgan, Ahmed \& Semeia, Abdellatif. (2020). Bandwidth-Oriented Allocation of GTS Slots for IEEE802.15.4.

IEEE Standard 802.15.4-2011, (2011), "IEEE Standard for Local and Metropolitan Area Networks," Part 15.4: Low-Rate Wireless Personal Area Networks (LR- WPANs).

Homera Durani (2018), Smart Home Automatic Applications using IoT with Blynk App, International Conference on Inventive Communication and Computational Technologies (ICICCT Galache, J.A. \& Sotres, Pablo \& Santana, Juan \& Gutierrez, Veronica \& Sánchez, Luis \& Munoz,

Luis. (2013). A Living Smart City: Dynamically Changing Nodes Behavior Through Over the Air Programming. Proceedings - 27th International Conference on Advanced Information Networking and Applications Workshops, WAINA 2013. 1271-1276. 10.1109/WAINA.2013.166. 


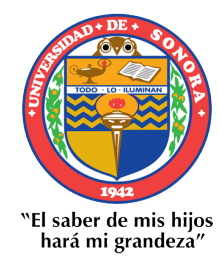

Año 13.

Núm. 33

https://revis
( Julio - Diciembre 2020)

Revista de Investigación

Académica sin Frontera

ISSN: 2007-8870

Recibido el 20 de junio de 2020. Dictaminado mediante arbitraje favorablemente 18 de diciembre de 2020.

2018), 2018 IEEE.

Maia, Guilherme \& Guidoni, Daniel \& Aquino, Andre \& Loureiro, Antonio. (2009). Improving an over-the-air programming protocol for wireless sensor networks based on small world concepts. 261-267. 10.1145/1641804.1641848.

S. C. Mukhopadhyay y H. Leung (2010) “Advances in wireless sensors and sensor networks”, Springer Science Business Media, 2010.

Quadri, S. \& Sidek, Othman. (2014). An Introduction to Over-the-Air Programming inWireless Sensor Networks. International journal of Computer Science \& Network Solutions. 2. 33-49.

Yogendra Singh Parihar, (2019) Internet of Things and Nodemcu A review of use of Nodemcu ESP8266 in IoT products, Journal of Emerging Technologies and Innovative Research (JETIR), vol. 6, no 06, pp. 1085-1088. 


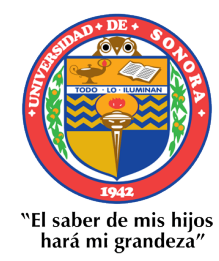

( Julio - Diciembre 2020)

Año 13.

Núm. 33
Revista de Investigación

Académica sin Frontera

ISSN: 2007-8870

Recibido el 20 de junio de 2020. Dictaminado mediante arbitraje favorablemente 18 de diciembre de 2020.

\section{Directorio Institucional}

Dr. Enrique Fernando Velázquez Contreras

Rector

Dr. Ramón Enrique Robles Zepeda

Secretario General Académico

Dra. Rosa María Montesinos Cisneros

Secretaria General Administrativa

Dr. Rodolfo Basurto Álvarez

Director de Vinculación y Difusión

Dra. Adriana Leticia Navarro Verdugo

Vicerrectora de la Unidad Regional Sur

Dr. Ernesto Clark Valenzuela

Director de la División de Ciencias Económicas y Sociales

Dr. Francisco Espinoza Morales

Secretario de la División de Ciencias Económico y Sociales

Dra. Leticia María González Velásquez

Jefe del Departamento de Ciencias Económico Administrativas

Dra. Lidia Amalia Zallas Esquer

Jefe de Departamento de Ciencias Sociales 


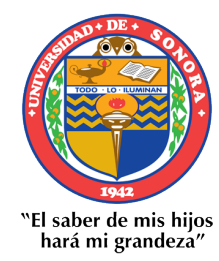

( Julio - Diciembre 2020)

Año 13.

Núm. 33
Revista de Investigación

Académica sin Frontera

ISSN: 2007-8870

Recibido el 20 de junio de 2020. Dictaminado mediante arbitraje favorablemente 18 de diciembre de 2020.

\title{
Comité Directivo
}

\author{
Editor Responsable \\ Dr. Francisco Espinoza Morales \\ Universidad de Sonora \\ Directora \\ Dra. Leticia María González Velásquez \\ Universidad de Sonora \\ Subdirector \\ Dr. Javier Carreón Guillen \\ Universidad Nacional Autónoma de México \\ Editor Científico \\ Dr. Cruz García Lirios \\ Universidad Autónoma del estado de México \\ Master Gráfico \\ M.T.I. Francisco Alan Espinoza Zallas \\ Universidad Estatal de Sonora
}

Nos complace anunciar que su diario, "Academic Research Journal Withoutborders" (ISSN/EISSN 2007-8870) fue evaluado positivamente en la indexación Citefactor, ahora la página de la revista está disponible en línea, en caso de cualquier problema.

Journals Master I International Innovative Journal Impact Factor (IIJIF)

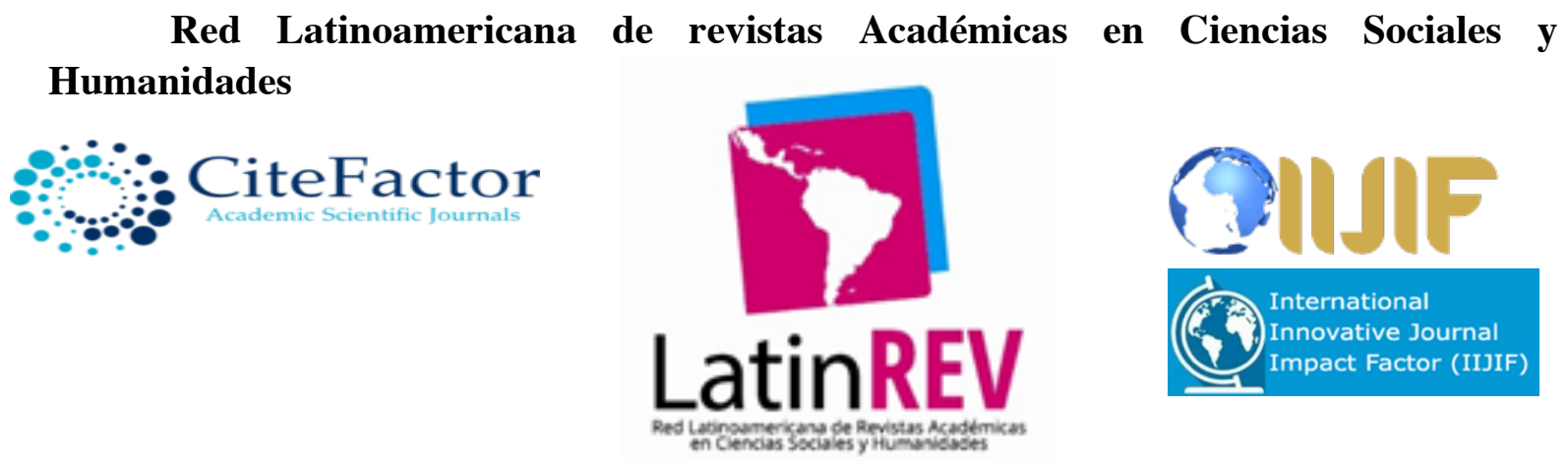




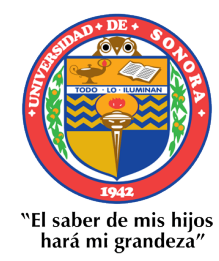

( Julio - Diciembre 2020)

Año 13.

Núm. 33
Revista de Investigación

Académica sin Frontera

ISSN: 2007-8870

Recibido el 20 de junio de 2020. Dictaminado mediante arbitraje favorablemente 18 de diciembre de 2020.

\section{Comité editorial}

Dra. Angélica María Rascón Larios

Universidad de Sonora. México

Dra. María del Rosario Molina González

Universidad de Sonora

Dra. Francisca Elena Rochin Wong

Universidad de Sonora. México

Dra. Lidia Amalia Zallas Esquer

Universidad de Sonora. México

Dra. Beatriz Llamas Arechiga

Universidad de Sonora. México

Dr. Rogelio Barba Álvarez

Universidad de Guadalajara. México

Dra. Rosa María Rincón Ornelas

Universidad de Sonora. México

Dr. Juan Flores Preciado

Universidad de Colima. México

Dr. Amado Olivares Leal. Universidad de Sonora

Universidad de Sonora. México

Dr. Guillermo Velázquez Valadez.

Instituto Politécnico Nacional (IPN) México

Dr. Hugo Nefstalí Padilla Torres.

Universidad Estatal de Sonora. México

Dr. Luis Ramón Moreno Moreno.

Universidad Autónoma de Baja California. México

Dr. Miguel Ángel Vázquez Ruiz.

Universidad de Sonora. México 


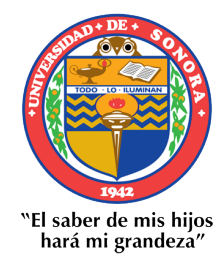

( Julio - Diciembre 2020)

Año 13.

Núm. 33
Revista de Investigación

Académica sin Frontera

ISSN: 2007-8870

https://revistainvestigacionacademicasinfrontera.unison.mx/index.php/RDIASF

Recibido el 20 de junio de 2020. Dictaminado mediante arbitraje favorablemente 18 de diciembre de 2020.

Dra. Lorena Vélez García.

Universidad Autónoma de Baja California. México

Dra. Pabla Peralta Miranda.

Universidad Simón Bolívar, Barranquilla, Colombia

Mtro. Roberto Espíritu Olmos

Universidad de Colima (FCA Tecomán) Colima

Dr. Héctor Priego Huertas.

Universidad de Colima (FCA Tecomán) Colima

Mtra. María Guadalupe Alvarado Ibarra.

Universidad de Sonora. México.

MSc. Celso Germán Sánchez Zayas

Universidad de Camagüey, Ignacio Agramonte Loynaz, Cuba

Dra. María Luisa Quintero Soto

Universidad Autónoma del Estado de México

Dr. Eyder Bolivar Mojica

Universidad Católica, Luis Amigó, Medellin, Colombia

Revisores de Textos en Inglés

Mtro. Renato Encinas

Mtra. Cecilia Guadalupe Martínez Solano

\section{Comité científico}

Dr. Rosendo Martínez Jiménez. Universidad Autónoma Benito Juárez de Oaxaca.

Dr. Hugo Neftalí Padilla. Universidad Estatal de Sonora

Dra. María Teresa Gaxiola Sánchez. Universidad de Sonora.

Dr. José Cesar Kaplan. Universidad Estatal de Sonora.

Dr. Alfredo Islas Rodríguez. Universidad de Sonora

Frecuencia de publicación: semestral / 2 números por año. 


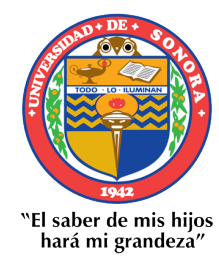

( Julio - Diciembre 2020)

Año 13.

Núm. 33

\section{Núm. 33}

Revista de Investigación

Académica sin Frontera

ISSN: 2007-8870

Recibido el 20 de junio de 2020. Dictaminado mediante arbitraje favorablemente 18 de diciembre de 2020.

Revista de Investigación Académica sin Frontera (RIASF) con (ISSN: 2007-8870) es un interlocutor internacional de acceso abierto revisado diario en línea en el ámbito del de las Ciencias Económicas Administrativas y Sociales. Su objetivo principal es dar a los trabajos de investigación de calidad. Cubre todas las sub-campos de los campos anteriormente mencionados. Proporciona la plataforma a académicos, estudiantes y profesionales. Sólo pública trabajos de investigación y artículos de revisión inicial. Documento presentado debe cumplir con algunos criterios como, debe ser original, inédita y no estén sometidos a ninguna otra revista.

RIASF es una revista arbitrada / Revisión por pares International. Publicamos documentos sobre una variedad de temas, contextos y estrategias de análisis que examinan la relación entre la rápida evolución para la Sociedad y la tecnología del conocimiento.

REVISTA DE INVESTIGACIÓN ACADÉMICA SIN FRONTERA, Año 13, No. 33, Julio - diciembre 2020, es una publicación semestral de investigación científica, editada por la Universidad de Sonora, a través de las División de Ciencias Económicas y Sociales, de la Unidad Regional Sur, Blvd. Lázaro Cárdenas No. 100, Col. Francisco Villa, Navojoa, Sonora, Sonora, México, C.P. 85880. Tel. (642) 42599-54.

https://revistainvestigacionacademicasinfrontera.unison.mx/index.php/RDIASF

revistaacademicasinfrontera@unison.mx.

Editor responsable: Francisco Espinoza Morales. Reserva de Derechos al Uso Exclusivo: 042013-121811323700-203 e ISSN: 2007-8870, ambos otorgados por el Instituto Nacional de Derecho de Autor. Inscrita en el Directorio de LATINDEX, con Núm. De folio 20014, folio único 14590. Responsable de la última actualización de este Número, Unidad Informática de la Universidad de Sonora, fecha de la última modificación, 30 de diciembre 2020, indexada a Cite Factor Academic Scientific Journal y Journals Master (IIJIF) y Red Latinoamericana de Revistas Académicas en Ciencias Sociales y Humanidades, (Latín Rev). Las opiniones expresadas por los autores no necesariamente reflejan la postura del editor de la publicación. Se autoriza la reproducción total o parcial de los contenidos e imágenes en la presente publicación siempre y cuando se cuente con la autorización del editor y se cite plenamente la fuente. 


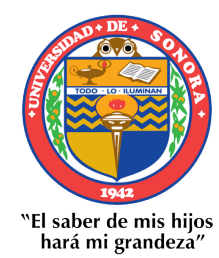

( Julio - Diciembre 2020)

Año 13.

Núm. 33
Revista de Investigación

Académica sin Frontera

ISSN: 2007-8870

Recibido el 20 de junio de 2020. Dictaminado mediante arbitraje favorablemente 18 de diciembre de 2020.

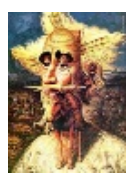

Nos complace anunciar que su diario, "Academic Research Journal Withoutborders" (ISSN/EISSN 2007-8870) fue evaluado positivamente en la indexación Citefactor, ahora la página de la revista está disponible en línea, en caso de cualquier problema.

Journals Master I International Innovative Journal Impact Factor (IJIF)

Red Latinoamericana de revistas Académicas en Ciencias Sociales y Humanidades
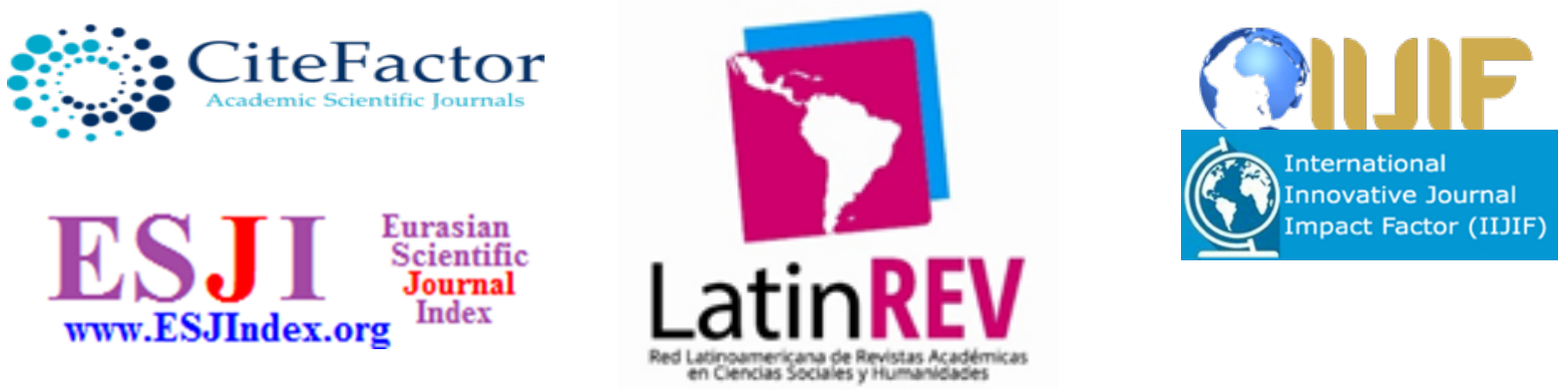

https://www.neliti.com
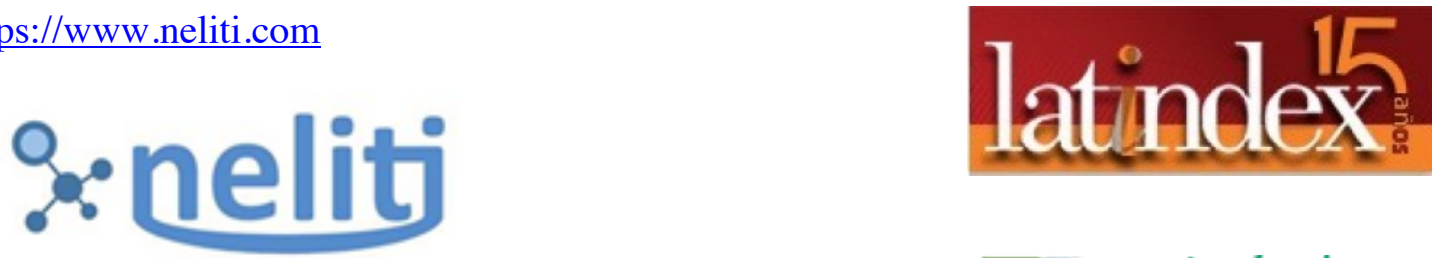

Indonesia's Research Repository

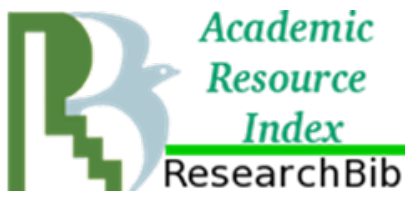

